\title{
The Effect of Contrast-enhanced Computed Tomography (CT) Scans on the Calculated Dose of Radiotherapy in a Thorax Phantom
}

\author{
Athar Ehtiati (iD) ${ }^{1}$, Payman Hejazi (iD 1, ${ }^{*}$, Mohsen Bakhshandeh $\mathbb{D i D}^{2}$, Ali Jabbary Arfaee ${ }^{3}$, Eftekhar Rajab \\ Bolookat $^{3}$, Majid Jadidi ${ }^{1}$ and Raheb Ghorbani ${ }^{4}$ \\ ${ }^{1}$ Department of Medical Physics, Semnan University of Medical Sciences, Semnan, Iran \\ ${ }^{2}$ Radiology Technology Department, Faculty of Paramedical Sciences, Shahid Beheshti University of Medical Sciences, Tehran, Iran \\ ${ }^{3}$ Department of Radiation Oncology, Shohada Tajrish Hospital, Tehran, Iran \\ ${ }^{4}$ Social Determinants of Health Research Center, Semnan University of Medical Sciences, Semnan, Iran \\ "Corresponding author: Department of Medical Physics, Semnan University of Medical Sciences, Semnan, Iran. Email: paymanhejazi@gmail.com
}

Received 2018 September 11; Revised 2020 October 16; Accepted 2020 October 18.

\begin{abstract}
Background: Despite the benefits of contrast-enhanced computed tomography (CT) scans in better tumor volume delineation, it can affect the accuracy of dose calculation in radiation therapy. This study examined this effect on a thorax phantom.

Objectives: The influence of different variables including the concentrations of the Visipaque contrast media, tumor sizes, and CT scan energies on the dose measurement was examined.

Methods: Transparent cylinders containing the contrast media were inserted in the lung area of the phantom and the CT scans were made. Non-enhanced CT scans were also acquired. Treatment planning using 2 opposite fields was performed on the CT scans and the doses were calculated in the treatment planning system. The results of the 2 sets of enhanced and non-enhanced CT scans were compared.

Results: The correlation between concentration and the percentage of mean dose of the tumor volume was significant in 2 of the tumor sizes. The differences in the mean doses of the 2 plans were examined and more than $3 \%$ increase was observed in higher concentrations of the contrast media.

Conclusions: According to this study, the suitable concentration of the contrast media administered and the CT scan energy should be considered. This would help to decrease the discrepancies between the calculated and delivered dose in radiotherapy treatments to a clinically acceptable level. The importance of time delays for CT scans after administration of the contrast media is emphasized.
\end{abstract}

Keywords: Radiotherapy, Lung Neoplasms, Contrast Media, Radiotherapy Dosage

\section{Background}

Lung neoplasms are the second most common cancer in both men and women and the leading cause of cancer death worldwide (1). Radiotherapy is one of the standard treatments for non-operable stages of lung neoplasms therapy and its accurate delivery is essential (2).

Due to the breathing movements of the thorax, a larger area is added to the Gross tumor volume (GTV), therefore accurate tumor volume delineation is important to decrease lung toxicity (3). Contrast-enhanced computed tomography (CT) scans can improve accurate delineation of the GTV and lymph nodes by distinguishing it from vascular structures. The thorax is a preferential site for examining the effect of contrast media due to it's numerous and large vascular structures (4). It is one of the 8 recommended tumor sites for administration of intravascular contrast as recommended by the Royal College of Radiologists (RCR) (5).

Despite all the benefits, based on contrast enhanced CT scans, the calculated dose in the treatment planning process might vary from the actual delivered dose because of the absent of the high-density contrast media during the radiotherapy treatment $(4,6)$. The fusion of 2 sets of CT scans, enhanced one for delineation and the nonenhanced one for treatment planning and monitor unit calculation might not be feasible because of the breathing motions of this region $((7,8)$. Furthermore Dual scanning if not necessary is preferred to be minimized according to as low as reasonably achievable (ALARA) principle (9).

Different investigations have been carried out on the effect of contrast media on the calculated dose of radiotherapy. Ramm et al. (10) examined this effect in a water 
phantom and found an increase in the calculated monitor unit (MU) depending on the concentration of the contrast media and the number of treatment fields. Robar et al. (11) found that the level of dose enhancement is related to the megavoltage energy used in radiotherapy. liauw et al. (6) found no significant variation in dose calculation for the head and neck region. Other investigations were also conducted on different anatomical regions and different techniques of radiotherapy and some of the results showed that the level of variations in the calculated dose was dependant on these factors $(7,12-14)$.

\section{Objectives}

The present study was performed on a thorax phantom to examine 4 variables, the first was the different concentrations of the visipaque contrast media to see which concentrations of the contrast media would lead to clinically acceptable changes in the calculated dose. The second was the effect of tumor size on the level of dose enhancement. The third was the probable difference in calculated dose variations at the time the contrast media is present in large vessels or is absorbed in the capillaries. The last was the influence of the CT scan energy and the impact that could have on the calculated dose in treatment planning.

\section{Methods}

\subsection{Phantom}

The phantom used in this study was made up of similar electron density materials to the actual structures of the human thorax (15). Including cork with a Hounsfield unit in the range of -774 to -841 HUs representing the Lungs, plexiglass of 80 to $171 \mathrm{HUs}$ as the soft tissues, and finally a dense fiber (phenolic resin cotton fiber rod) with the HU between 565 and 717 HUs instead of the vertebral bones. It is worth mentioning that the range of HUs presented above are obtained from our treatment planning system (TPS) software. The dimension of this phantom was based on intensity-modulated radiotherapy (IMRT) thorax phantoms (Figure 1).

The attendance of contrast media was investigated in 2 situations, firstly in large blood vessels and next in the capillaries. The presence of contrast media in large blood vessels was tested by inserting transparent cylinders with a diameter of $1 \mathrm{~cm}$ in the range of actual large blood vessels near the mediastinum, containing the contrast media.

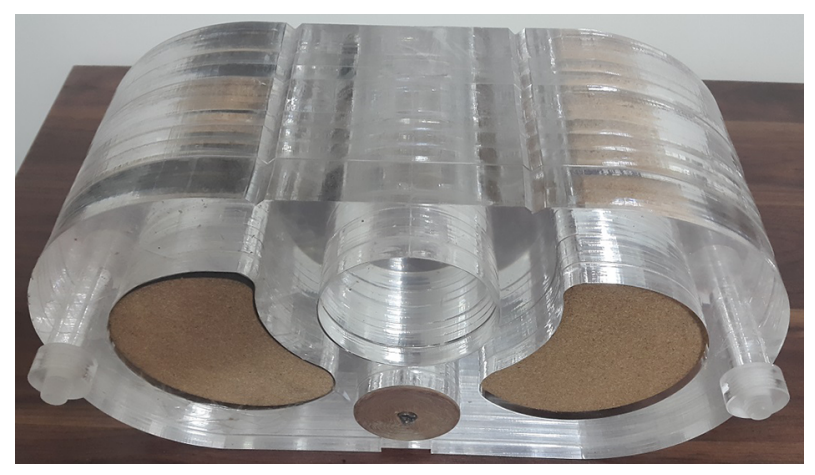

Figure 1. The thorax phantom used in this study

The contrast media used in this study was visipaque (iodixanol) containing $320 \mathrm{mgI} / \mathrm{mL}$ in a $50 \mathrm{ml}$ vial, manufactured by GE healthcare, Ireland (16). Five different concentrations of Visipaque were used by diluting it in 5 steps in normal saline solution simulating the dilution of contrast media in blood flowing through the vessels.

In order to demonstrate the uptake of contrast media by the capillaries after a complete blood circulation, the cork slices were soaked in a tank of visipaque with a concentration of $1 / 48$ of the initial concentration.

\subsection{CT Acquisition}

The phantom was scanned in the 16-slice SOMATOM Emotion, Siemens CT scanner with 3 different CT scan energies of 80,110 , and $130 \mathrm{kVp}$. The exposure time was $600 \mathrm{~ms}$ and slice thickness was $5 \mathrm{~mm}$. Different series of contrastenhanced CT scans were acquired by changing the cylinders containing different concentrations and no contrast CT scans were acquired for the 3 different kilo voltages.

\subsection{Treatment Planning}

The CT scans were imported to the Eclipse V13.1 software manufactured by Varian Medical System for the Clinac 600c-6x linear accelerator. Eclipse is a comprehensive treatment planning system, which facilitated the treatment planning and optimization of this study through its anisotropic analytical algorithm (AAA) photon dose calculation model. This model is a 3D pencil beam convolutionsuperposition algorithm, which provides a fast and accurate dose calculation for clinical photon beams even in complex tissue heterogeneities like lungs (17).

Three sizes of tumors representing different stages of the disease were delineated (Figure 2) near the mediastinum on contrast-enhanced with different concentrations and non-enhanced CT scans. 

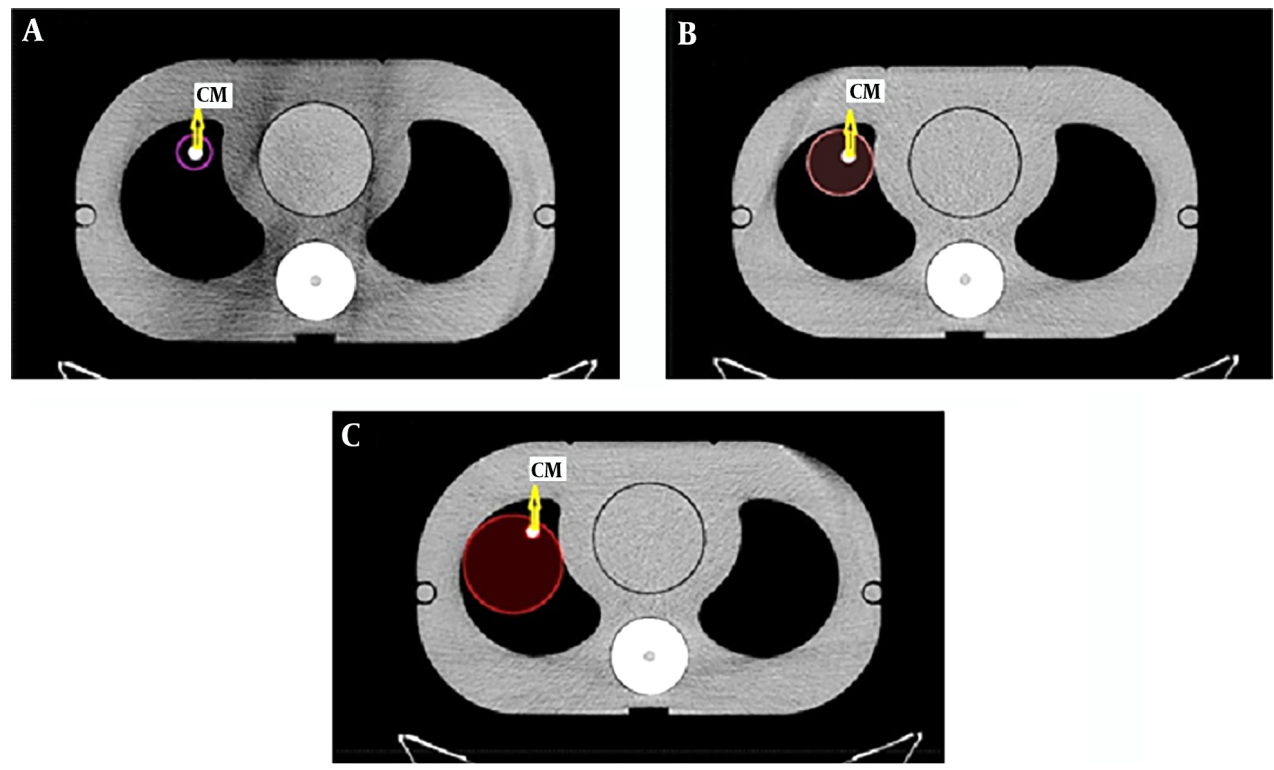

Figure 2. The transversal view of the position of the tumors and the Visipaque contrast media (CM) within each tumor, A, representing the $2.5 \mathrm{~cm}$ GTV (purple); B the $5 \mathrm{~cm}$ GTV (pink); and C, the $7.5 \mathrm{~cm}$ GTV (red) of tumors contours.

In the contrast-enhanced plans, the GTV contained the cylinders with contrast media but in the non-enhanced plans, the GTV was contoured in a way that only the cork material representing the lung tissue was delineated. The mediastinum, lungs, and spinal cord were contoured as the organs at risk.

The volume ratio of the contrast media over the GTV in the $2.5 \mathrm{~cm}$ tumor size was 0.244 , in the $5 \mathrm{~cm}$ tumor size it was 0.045 , and it was 0.02 for the $7.5 \mathrm{~cm}$ tumor size. The ratios were acquired from the formula of the cylinder volume for the volume of the contrast media over the volumes that the TPS calculates for any specific contour. Figure 3 shows this concept more illustrative

The treatment planning was performed on the CT scans using 2 opposing fields, anterior-posterior and posterioranterior with equal beam weights. The energy used was $6 \mathrm{MV}$ photon beams and the reference point with $100 \%$ of the daily dose of $2 \mathrm{~Gy}$ was adjusted on the isocenter of the 2 fields.

The monitor units (MUs) of each field, the mean dose of the GTV, and the line dose profile for the contrast and no contrast plans were calculated through the anisotropic analytical algorithm V13.0.26 with the calculation grid size of $0.25 \mathrm{~cm}$. For each CT scan energy, a specific CT calibration table was used for CT number to density conversion.

\subsection{Analysis}

The analysis of the data obtained from this study was performed by the Pearson correlation test in order to find the probable correlation through the SPSS 16 software.

\section{Results}

The dose line profile of the midline passing through the reference point for the no contrast plan (Figure $4 \mathrm{~A}$ ) with the tumor size of $2.5 \mathrm{~cm}$ and the CT scan energy of 130 $\mathrm{kVp}$ was calculated with the Eclipse 013 treatment planning system. It was compared with the dose line profile of the contrast plan with $1 / 3$ of the initial concentration (Figure $4 \mathrm{~B})$. The red vertical line illustrated in Figure 5 was the midline passing through both the reference point and the contrast media. The total dose line profile as well as the dose line profile in each field are demonstrated in Figure 4A and B.

Figure 5 shows that the photon beam passes through the soft tissue and then the right lung, after that the contrast media and then the isocenter(reference point). In Figure 4 , the dose line profiles with and without the contrast media and passing all these mediums is illustrated. The dose of $100 \%$ is applied to the isocenter. A peak with a maximum dose of $114 \%$ is observed exactly where the contrast media exists. The peak was also observed in the 4 other concentrations examined in this study. 

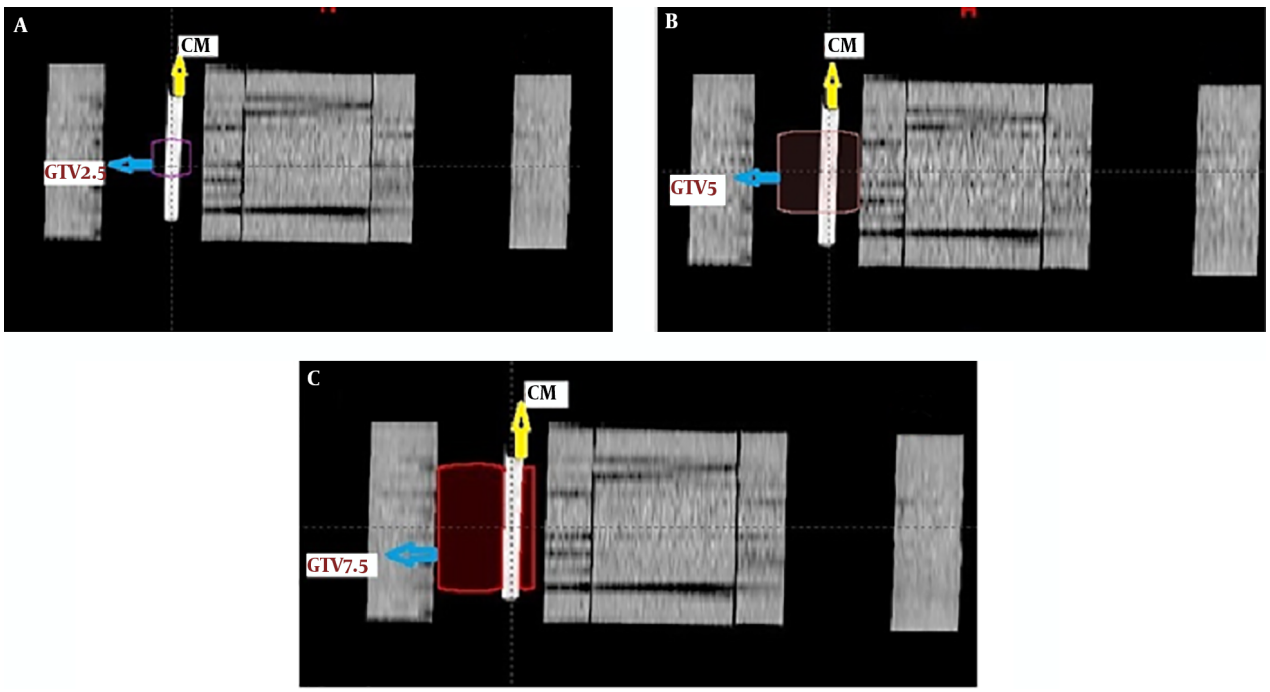

Figure 3. The frontal view showing the contrast media cylinder (CM) within each tumor A, showing contours of the $2.5 \mathrm{~cm}$ GTV; B, the $5 \mathrm{~cm}$ GTV; and C, the $7.5 \mathrm{~cm}$ GTV.

The Pearson correlation test was made to acquire the Pearson coefficients and to test the significance of the correlation between the GTV mean dose and concentration of the contrast media. Two variables of tumor size and CT scan energies were considered (Table 1 ).

According to Table 1, in most of the cases except for the tumor size of $7.5 \mathrm{~cm}$, the correlation was significant at the 0.05 level (18).

The clustered column charts of the variations of the mean dose in different concentrations of the contrast media are illustrated in Figures 6 and 7. First, changing the CT scan energies in constant tumor sizes (Figure 6) and then changing the tumor sizes in constant CT scan energies are showed in Figure 7.

The percentage of mean dose differences between the contrast-enhanced plans and non-enhanced plans in all the cases is calculated in Table 2.

According to Table 2, as the concentration raised, the mean dose differences increased, so that in 1/3 of the initial concentration, the mean dose differences raised above $3 \%$. In bigger tumor sizes, the mean dose differences remained below $3 \%$. In the case of comparing the absorption of the capillaries with the no contrast plan in the 3 energies and tumor sizes, the maximum difference in the mean dose was $0.8 \%$.

The monitor unit of each field was calculated through the treatment planning system for all the concentrations of the contrast media starting from the concentration of 0 (no contrast plan) in different tumor sizes. Table 3 shows the calculated monitor units in the anterior-posterior (AP) field.

Table 3 shows an increase in monitor units as the concentration increased in most of the cases and adversely a decrease was observed as the tumor size increased in the same concentration. The posterior-anterior (PA) field was not mentioned because it was almost constant in all concentrations.

\section{Discussion}

In the treatment planning process of radiotherapy, contrast media may be administered in CT scanning in order to assist for accurate tumor and organ at risk delineation. However, the absence of the contrast media during the treatment might affect the accuracy of dose calculations. This effect was examined in this study.

International Commission on Radiation Units and Measurements (ICRU) recommends that the delivered dose should not deviate $-{ }^{+} 5 \%$ from the prescribed dose. Due to various probable uncertainties in dose delivery to patients, more than 3\% accuracy is required in each step (19). Therefore, we considered 3\% and above as a significant clinical dose discrepancy in dose calculations. According to the results, differences between the mean doses of the contrast-enhanced plans and non-enhanced plans showed an increase in the calculated dose of the contrast plans. However, the differences were only clinically significant at high concentrations of the visipaque contrast media (1/3 of the initial concentration) in the CT scan energy of 130 
A
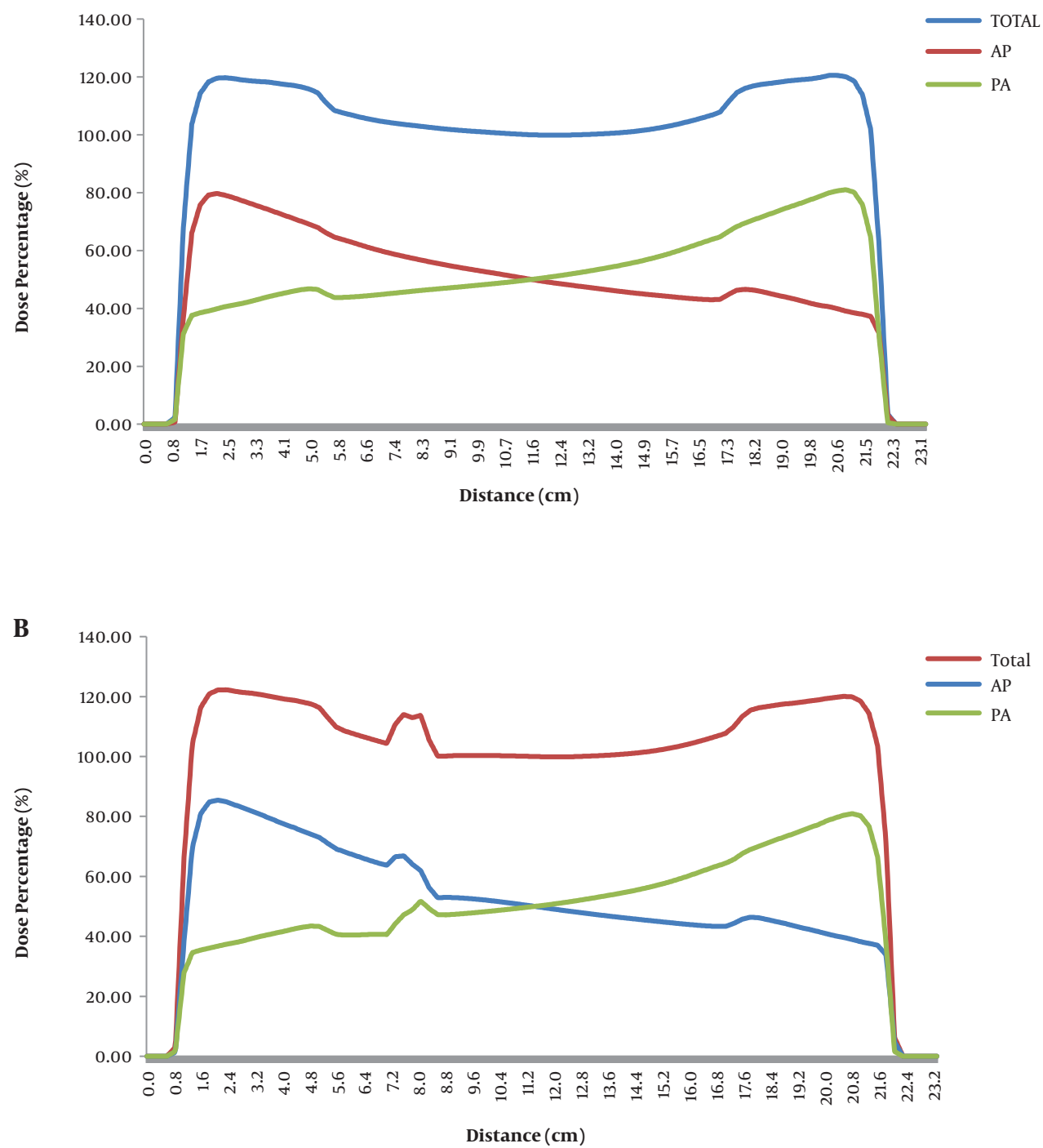

Figure 4. A, The dose line profile of the non-contrast plan; and B, the contrast plan. AP stands for Anterior-Posterior field and PA for the posterior-Anterior field.

\begin{tabular}{|c|c|c|c|c|c|c|}
\hline \multirow{2}{*}{ Tumor Size, cm } & \multicolumn{2}{|c|}{ Energy of $130 \mathrm{kVp}$} & \multicolumn{2}{|c|}{ Energy of $110 \mathrm{kVp}$} & \multicolumn{2}{|c|}{ Energy of $80 \mathrm{kVp}$} \\
\hline & $\mathbf{r}$ & P-Value & $\mathbf{r}$ & P-Value & $\mathbf{r}$ & P-Value \\
\hline 2.5 & 0.910 & $0.012^{\mathrm{b}}$ & 0.928 & $0.008^{\mathrm{b}}$ & 0.938 & $0.006^{\mathrm{b}}$ \\
\hline 5 & 0.844 & $0.035^{\mathrm{b}}$ & 0.970 & $0.001^{b}$ & 0.954 & $0.003^{b}$ \\
\hline 7.5 & 0.381 & 0.456 & 0.739 & 0.093 & 0.764 & 0.077 \\
\hline
\end{tabular}

${ }^{\mathrm{a}}$ Letter $\mathrm{r}$ indicates the Pearson correlation coefficient

${ }^{b}$ Used when the P-value $<0.05$, which means the correlation is significant at the 0.05 level.

and $110 \mathrm{kVp}$. With decreasing CT scan energy (80 kV energy), the mean dose differences raised to a maximum of
$5.3 \%$, which is higher than the overall dose delivery uncertainties accepted by the ICRU. Even 1/12 of the concentra- 


\begin{tabular}{|c|c|c|c|c|c|c|c|c|c|}
\hline \multirow{3}{*}{$\begin{array}{l}\text { The Concentration } \\
\text { Compared with no } \\
\text { Contrast }\end{array}$} & \multicolumn{9}{|c|}{$\frac{D_{C+}-D_{c_{0}}}{D_{c_{0}}}$} \\
\hline & \multicolumn{3}{|c|}{ Energy of $130 \mathrm{kVp}, \%$} & \multicolumn{3}{|c|}{ Energy of $110 \mathrm{kVp}$, \% } & \multicolumn{3}{|c|}{ Energy of $80 \mathrm{kVp}$, \% } \\
\hline & $\mathrm{T}=2.5 \mathrm{~cm}$ & $\mathrm{~T}=5 \mathrm{~cm}$ & $\mathrm{~T}=7.5 \mathrm{~cm}$ & $\mathrm{~T}=2.5 \mathrm{~cm}$ & $\mathrm{~T}=5 \mathrm{~cm}$ & $\mathrm{~T}=7.5 \mathrm{~cm}$ & $\mathrm{~T}=2.5 \mathrm{~cm}$ & $\mathrm{~T}=5 \mathrm{~cm}$ & $\mathrm{~T}=7.5 \mathrm{~cm}$ \\
\hline Capillaries & 0.1 & 0.3 & 0.8 & 0.2 & 0.3 & 0.6 & 0.1 & 0.1 & 0.3 \\
\hline $1 / 48$ & 1.3 & 0.1 & 0.8 & 1.9 & 0.6 & 0 & 2.3 & 1.2 & 0 \\
\hline $1 / 24$ & 1.8 & 0.9 & 0.7 & 2.4 & 1.3 & 0.6 & 2.5 & 1.2 & 0.2 \\
\hline 1/12 & 1.7 & 0.7 & 0.6 & 2.6 & 1.7 & 0.3 & 2.9 & 1.8 & 0.1 \\
\hline $1 / 6$ & 1.9 & 0.7 & 0.5 & 2.8 & 1.6 & 0.9 & $3.4^{\mathrm{b}}$ & 2 & 0.4 \\
\hline $1 / 3$ & $3_{3}^{b}$ & 1 & 0.6 & $4.2^{\mathrm{b}}$ & 2.5 & 0.5 & $5.1^{\mathrm{b}}$ & 2.6 & 0.6 \\
\hline
\end{tabular}

Abbreviation: T, tumor size.

a $\frac{D_{C+}-D_{c_{0}}}{D_{c_{0}}}$ is the percentage dose difference between the contrast-plan and no contrast-plan.

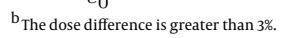

Table 3. Calculated Monitor Units of the Anterior-Posterior Field in Different Tumor Sizes, Concentrations, and CT Scan Energies

\begin{tabular}{|c|c|c|c|c|c|c|c|c|c|}
\hline \multirow{3}{*}{ Concentration } & \multicolumn{9}{|c|}{ The Calculated Monitor Unit } \\
\hline & \multicolumn{3}{|c|}{ Energy of $130 \mathrm{kVp}$} & \multicolumn{3}{|c|}{ Energy of $110 \mathrm{kVp}$} & \multicolumn{3}{|c|}{ Energy of $80 \mathrm{kVp}$} \\
\hline & $\mathrm{T}=2.5 \mathrm{~cm}$ & $T=5 \mathrm{~cm}$ & $\mathrm{~T}=7.5 \mathrm{~cm}$ & $\mathrm{~T}=2.5 \mathrm{~cm}$ & $\mathrm{~T}=5 \mathrm{~cm}$ & $\mathrm{~T}=7.5 \mathrm{~cm}$ & $\mathrm{~T}=2.5 \mathrm{~cm}$ & $\mathrm{~T}=5 \mathrm{~cm}$ & $\mathrm{~T}=7.5 \mathrm{~cm}$ \\
\hline $\mathbf{0}$ & 130 & 124 & 115 & 130 & 124 & 115 & 130 & 124 & 114 \\
\hline $1 / 48$ & 133 & 125 & 115 & 133 & 125 & 115 & 133 & 126 & 115 \\
\hline 1/24 & 134 & 126 & 115 & 133 & 126 & 117 & 134 & 126 & 115 \\
\hline 1/12 & 134 & 127 & 115 & 134 & 128 & 115 & 135 & 127 & 115 \\
\hline $1 / 6$ & 134 & 127 & 115 & 136 & 127 & 116 & 138 & 128 & 116 \\
\hline $1 / 3$ & 140 & 130 & 117 & 142 & 131 & 117 & 146 & 132 & 118 \\
\hline
\end{tabular}

Abbreviation: $T$, of the tumor size.

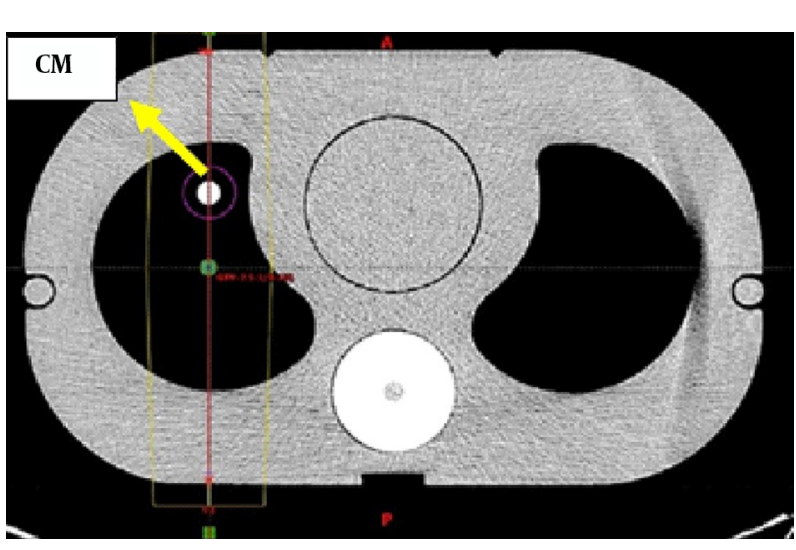

Figure 5. The CT scan of the phantom with the red midline passing through the green reference point (isocenter) and the contrast media (CM) in the contrast plan.

tion shows clinically significant differences. The different result in dose calculation for different energies is related to the predominance of the photoelectric effect at $80 \mathrm{kVp}$ energy.

As mentioned, the dose differences were found to be statistically significant but in most cases were clinically acceptable, this finding is in line with the study conducted by Xiao et al. (7) In another study by Lees et al. (4) no clinically significant differences were reported, and in a study by Kimlin et al. (8) the results were also considered negligible. The minimal impact of the presence of contrast media on dose calculation was observed in some studies which were depended on the tumor sites and the number of treatment fields, as it was predicted by Ramm et al. (10), especially in the head and neck cancers because of small blood vessels and treatments with high numbers of fields such as IMRT technique $(12,14,20)$.

The increase in tumor size shows fewer discrepancies between the mean doses of the contrast-enhanced plans and the no contrast ones. The ratio of the volume of contrast media over the volume of the GTV for the tumor size of $2.5 \mathrm{~cm}$ was 0.244 , for the $5 \mathrm{~cm}$ tumor size was 0.045 , and for the $7.5 \mathrm{~cm}$ diameter of the tumor was 0.02 . As we know, the mean dose is the average dose of the calculation grids defined in a volume. As the tumor size grows, the volume with a high density compared to the normal density of the lungs, decreases and the average dose consequently decreases.

The monitor unit calculation showed an increase as the concentration of the contrast media increases and it approves the results of the studies of Rankine et al. (21) and 

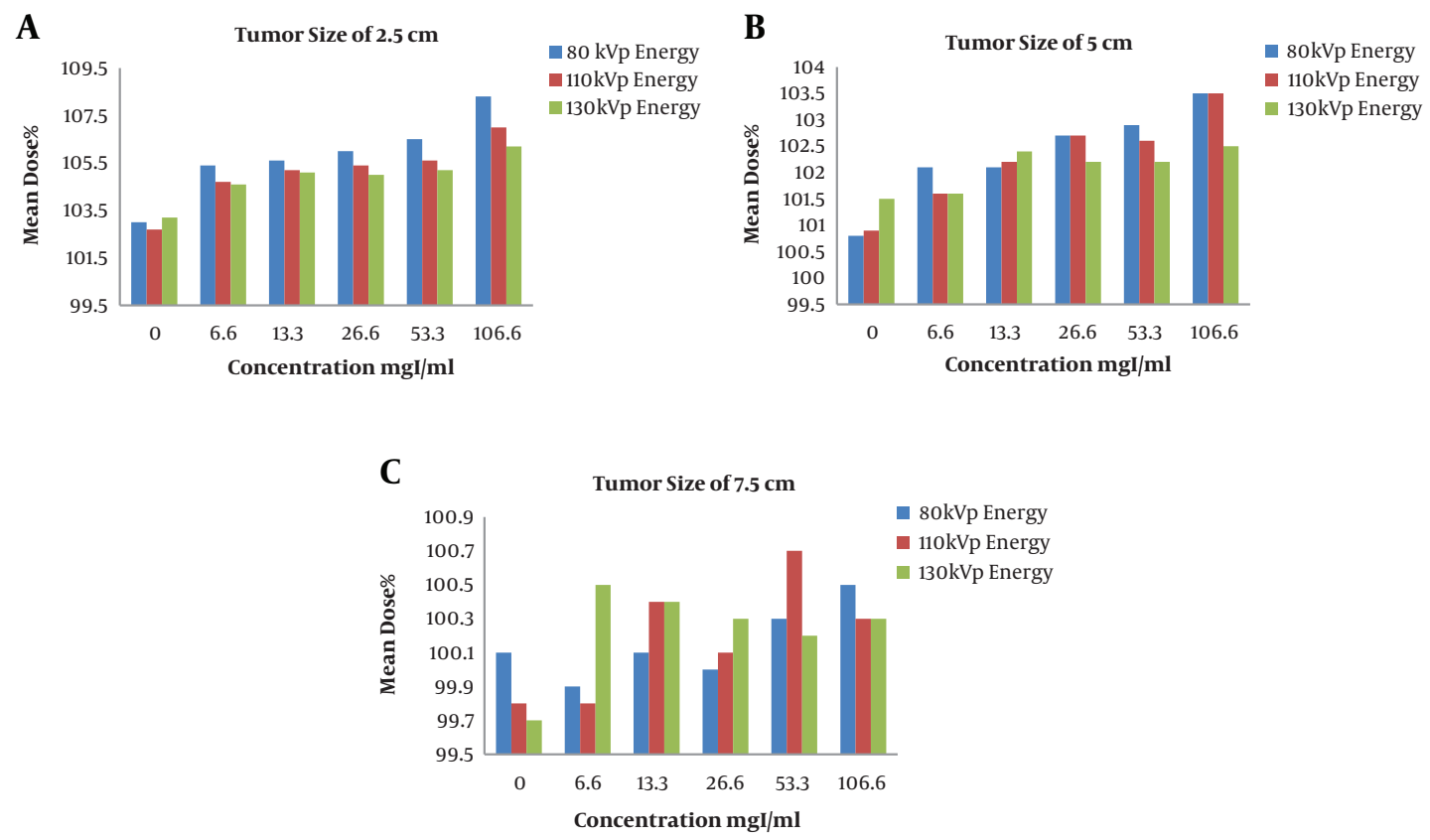

Figure 6. The variations of the mean dose in different concentrations, by changing the CT scan energies in constant tumor sizes: A, $2.5 \mathrm{~cm}$; B, $5 \mathrm{~cm}$; C, $7.5 \mathrm{~cm}$.
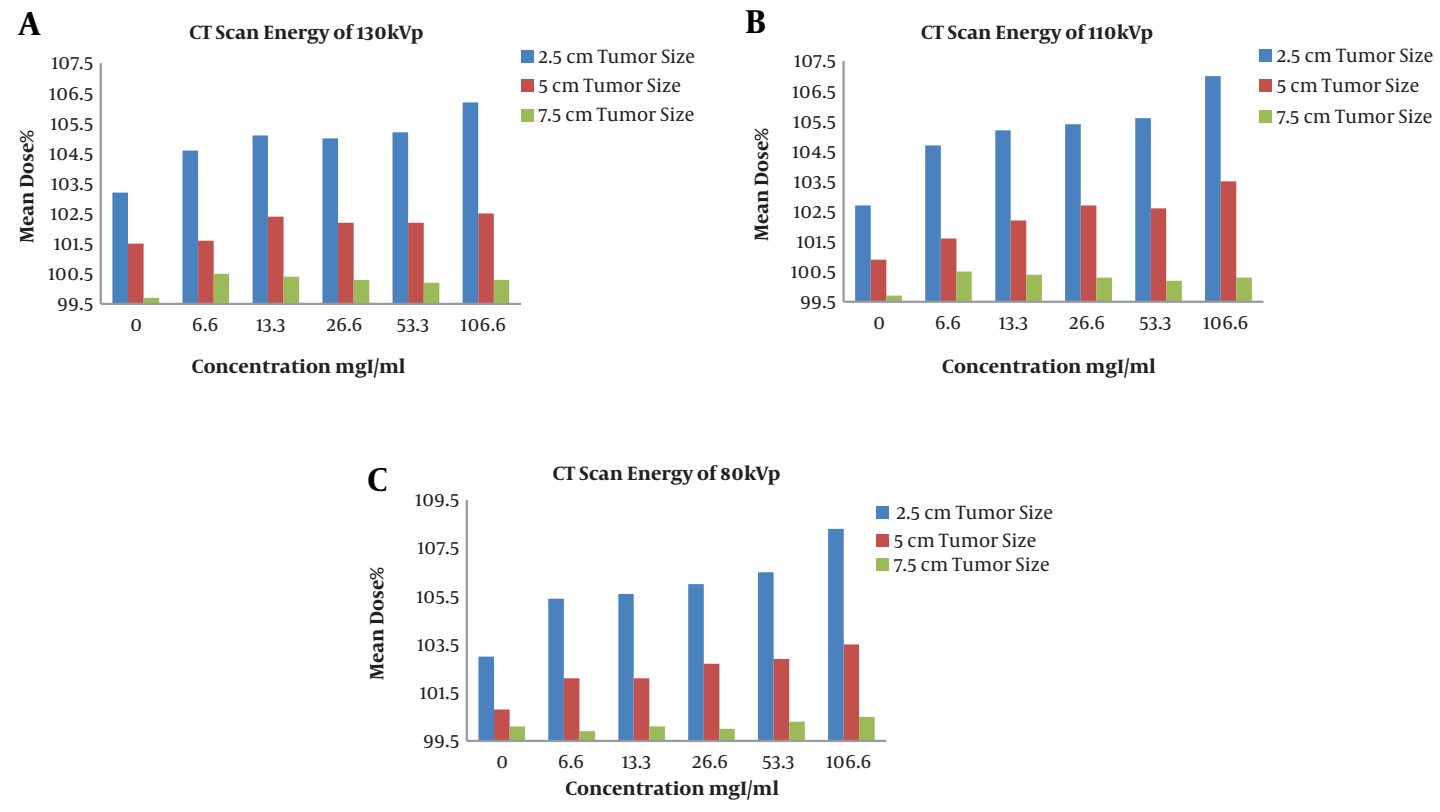

Figure 7. The variations of the mean dose in different concentrations, by changing the tumor sizes in constant CT scan energies: A, $130 \mathrm{kVp}$; B, $110 \mathrm{kVp}$;, $80 \mathrm{kVp}$.

Ramm et al. (10). Comparing the contrast plans and no contrast plans, an increase in MUs was observed as it was for the studies of Burridge et al. and Jabbari et al. for the oesophageal region $(3,22)$.

\subsection{Conclusions}

According to the findings of this study, increasing the concentration of the contrast media and decreasing the CT scan energy would result in an increase in the calculated 
dose and MU by the TPS. We concluded that the suitable concentration of the contrast media administered and the CT scan energy should be considered. This would help to decrease the discrepancies between the calculated and delivered doses in radiotherapy treatments to a clinically acceptable level.

The different concentration assessment in this study demonstrates the dilution of the contrast media due to the blood circulation throughout the patient's body. This study shows the importance of time delays for CT scans after administration of the contrast media. We recommend the maximum administered concentration of the visipaque contrast media to be $1 / 6$ of the initial concentration at the time of scanning with utilizing higher CT scan energies, $110 \mathrm{kVp}$, and above. This practical conclusion can also help to prevent an unwanted increase in the linear accelerator workload due to the increase in MUs.

\section{Footnotes}

Authors' Contribution: Study concept and design: Payman Hejazi. Acquisition of data: Athar Ehtiati. Analysis and interpretation of data: Athar Ehtiati, Payman Hejazi, and Mohsen Bakhshandeh. Drafting of the manuscript: Athar Ehtiati. Critical revision of the manuscript for important intellectual content: Payman Hejazi. Statistical analysis: Athar Ehtiati, Payman Hejazi, and Raheb Ghorbani. Administrative, technical, and material support: Payman Hejazi, Mohsen Bakhshandeh, Ali Jabbary Arfaee, Eftekhar Rajab Bolookat, and Majid Jadidi. Study supervision: Payman Hejazi.

Conflict of Interests: The authors certify that they have no affiliations with or involvement in any organization or entity with any financial interest in the subject matter or materials discussed in this manuscript. All the authors declare that they have no conflict of interests.

Funding/Support: The grant of the research was supported by Semnan University Of Medical Sciences.

\section{References}

1. Molina JR, Yang P, Cassivi SD, Schild SE, Adjei AA. Non-small cell lung cancer: epidemiology, risk factors, treatment, and survivorship. Mayo Clin Proc. 2008;83(5):584-94. doi: 10.4065/83.5.584. [PubMed: 18452692]. [PubMed Central: PMC2718421].

2. Baumann M, Petersen C. TCP and NTCP: a basic introduction. Rays 2005;30(2):99-104. [PubMed:16294901]

3. Burridge NA, Rowbottom CG, Burt PA. Effect of contrast enhanced CT scans on heterogeneity corrected dose computations in the lung. J Appl Clin Med Phys. 2006;7(4):1-12. doi: 10.1120/jacmp.v7i4.2240. [PubMed: 17533351]. [PubMed Central: PMC5722395].
4. Lees J, Holloway L, Fuller M, Forstner D. Effect of intravenous contrast on treatment planning system dose calculations in the lung Australas Phys Eng Sci Med. 2005;28(3):190-5. doi: 10.1007/BF03178715. [PubMed: 16250475].

5. Royal College of Radiologists . Faculty of Clinical Oncology Board Imaging for Oncology: Collaboration Between Clinical Radiologists and Clinical Oncologists in Diagnosis, Staging and Radiotherapy Planning. Royal College of Radiologists; 2004.

6. Liauw SL, Amdur RJ, Mendenhall WM, Palta J, Kim S. The effect of intravenous contrast on intensity-modulated radiation therapy dose calculations for head and neck cancer. Am J Clin Oncol. 2005;28(5):456-9. doi:10.1097/01.coc.0000170796.89560.02. [PubMed: 16199983].

7. Xiao J, Zhang H, Gong Y, Fu Y, Tang B, Wang S, et al. Feasibility of using intravenous contrast-enhanced computed tomography (CT) scans in lung cancer treatment planning. Radiother Oncol.2010;96(1):73-7. doi: 10.1016/j.radonc.2010.02.029. [PubMed: 20347496].

8. Kimlin K, Mitchell J, Knight RT. Effects of iodinated contrast media on radiation therapy dosimetry for pathologies within the thorax. Radiographer. 2006;53(2):30-4. doi:10.1002/j.2051-3909.2006.tb00053.x.

9. Williams K, Probst $\mathrm{H}$. Use of IV contrast media in radiotherapy planning CT scans: A UK audit. Radiography. 2016;22:S28-32. doi: 10.1016/j.radi.2016.06.006.

10. Ramm U, Damrau M, Mose S, Manegold KH, Rahl CG, Bottcher HD. Influence of CT contrast agents on dose calculations in a 3D treatment planning system. Phys Med Biol. 2001;46(10):2631-5. doi: 10.1088/00319155/46/10/308. [PubMed: 11686279].

11. Robar JL, Riccio SA, Martin MA. Tumour dose enhancement using modified megavoltage photon beams and contrast media. Phys Med Biol. 2002;47(14):2433-49. doi: 10.1088/0031-9155/47/14/305. [PubMed: 12171332].

12. Choi Y, Kim JK, Lee HS, Hur WJ, Hong YS, Park S, et al. Influence of intravenous contrast agent on dose calculations of intensity modulated radiation therapy plans for head and neck cancer. Radiother Oncol. 2006;81(2):158-62. doi: 10.1016/j.radonc.2006.09.010. [PubMed: 17050020].

13. Lee FK, Chan CC, Law CK. Influence of CT contrast agent on dose calculation of intensity modulated radiation therapy plan for nasopharyngeal carcinoma. J Med Imaging Radiat Oncol. 2009;53(1):114-8. doi: 10.1111/j.1754-9485.2009.02046.x. [PubMed: 19453537].

14. Shibamoto Y, Naruse A, Fukuma H, Ayakawa S, Sugie C, Tomita N. Influence of contrast materials on dose calculation in radiotherapy planning using computed tomography for tumors at various anatomical regions: a prospective study. Radiother Oncol. 2007;84(1):52-5. doi 10.1016/j.radonc.2007.05.015. [PubMed: 17532496].

15. Bridge P. CT Anatomy for Radiotherapy. M\&K; 2011.

16. Iodixanol. Wikipedia, The Free Encyclopedia; 2020. Available from: https://en.wikipedia.org/w/index.php?title=Iodixanol\&oldid= 937544212.

17. Sievinen J, Ulmer W, Kaissl W. AAA photon dose calculation model in Eclipse. Palo Alto (CA). 2005;118:2894.

18. Chaikh A, Giraud JY, Perrin E, Bresciani JP, Balosso J. The choice of statistical methods for comparisons of dosimetric data in radiotherapy. Radiat Oncol. 2014;9:205. doi: 10.1186/1748-717X-9-205. [PubMed: 25231199]. [PubMed Central: PMC4261592].

19. Sellakumar P, Arun C, Sanjay SS, Ramesh SB. Comparison of monitor units calculated by radiotherapy treatment planning system and an independent monitor unit verification software. Phys Med 2011;27(1):21-9. doi:10.1016/j.ejmp.2010.01.006. [PubMed: 20172756].

20. Letourneau D, Finlay M, O'Sullivan B, Waldron JN, Cummings BJ, Ringash J, et al. Lack of influence of intravenous contrast on head and neck IMRT dose distributions. Acta Oncol. 2008;47(1):90-4. doi 10.1080/02841860701418861. [PubMed: 17934894] 
21. Rankine AW, Lanzon PJ, Spry NA. Effect of contrast media on megavoltage photon beam dosimetry. Med Dosim. 2008;33(3):169-74. doi: 10.1016/j.meddos.2007.04.007. [PubMed: 18674680].

22. Nasrollah J, Mikaeil M, Omid E, Mojtaba SS, Ahad Z. Influence of the intravenous contrast media on treatment planning dose calculations of lower esophageal and rectal cancers. J Cancer Res Ther. 2014;10(1):147-52. doi: 10.4103/0973-1482.131465. [PubMed: 24762502]. 